Correo: innova@uide.edu.ec

\title{
Diseño e Implementación de máquina cargadora frontal sobre ruedas L120E Volvo para la limpieza y remoción de tierra por deslaves en la vía Aloag-Santo Domingo
}

\section{Design and Implementation of Volvo L120E front wheel loader machine for cleaning and removal of dirt by landslides in the Aloag-Santo Domingo road}

\author{
Marcelo González \\ Marco Noroña \\ Universidad Internacional del Ecuador, Ecuador
}

Autor para correspondencia: manoroname@internacional.edu.ec, magonzalezto@internacional.edu.ec

Fecha de recepción: 08 de Septiembre de 2016 - Fecha de aceptación: 01 de Noviembre de 2016

Resumen: A continuación resumiré el contenido de este artículo científico, en donde describo cual es el diseño e implementación de una cargadora frontal sobre ruedas de la marca volvo, que es empleada para la remoción de tierras que caen en os taludes de la vía Aloag-Santo domingo, como sabemos esta es una carretera muy transitada, y cada cierto tiempo se presenta caída de tierra y piedras debido al talud formado por la montañas y al constante desprendimiento de tierra que se produce en la montañas ya sea por fallas geológicas o por el clima. Dentro de los conceptos y procedimientos que detallo en este artículo, está la definición de lo que es un talud, como se realiza la correcta remoción de la tierra, y las características principales de la cargadora frontal de la marca Volvo. Indicare los métodos seleccionados para a ampliación del cargador frontal en esta obra de remoción de tierras, así como el proceso llevado a cabo para el modelado gráfico 3D y el diseño del dibujo 2D. Por último daremos los resultados y discusión de la implementación del cargador frontal, y daré mi concusión sobre el tema expuesto en este artículo.

Palabras claves: cargador; talud; tierra; diseño; carretera

\begin{abstract}
Then summarize the contents of this scientific paper, where I describe what the design and implementation of a front wheel loader from Volvo, which is used for the removal of land falling on you slope of the road Aloag Santo Domingo as we know this is a busy road, and every so often falling dirt and rocks occurs because of the slope formed by the mountains and the constant landslide that occurs in the mountains either by geological faults or climate. Within the concepts and procedures detailed in this article, it is the definition of what a batter, as the correct soil removal is performed, and the main characteristics of the front loader Volvo. NOTED the methods selected for a front loader expansion in this work of earth moving and the process undertaken for 3D graphical modeling and design of 2D drawing. Finally we give the results and discussion of the implementation of the front loader, and give my concussion on the subject matter of this article.

Key words: charger; slope; land; design; road
\end{abstract}




\section{Introducción}

Los deslizamientos de laderas, desprendimientos de rocas y aludes de nieve son algunos de los procesos geológicos más comunes en la superficie de la Tierra. Forman parte del ciclo natural del terreno ya que la erosión y la gravedad actúan constantemente para transportar materiales de las zonas más altas hacia abajo.

\section{Deslaves}

Es la caída de rocas o tierra desde una ladera, en forma lenta o rápida, que se produce en épocas de lluvia o a causa de un sismo. Dependiendo de la magnitud, destruye todo lo que se encuentra a su paso. En la costa, sierra y en la región oriental ocurren deslizamientos porque Ecuador es un país montañoso. La mayoría se presenta durante las estaciones lluviosas. Cuando el suelo recibe una gran cantidad de agua, la tierra se ablanda y se desprende formando flujos de lodo, que se precipitan pendiente a bajo.

Algunas personas contribuyen a que ocurran deslizamientos, cuando construyen con materiales pesados en terrenos débiles, o cuando realizan excavaciones que desestabilizan las laderas. La deforestación también es una causa de los deslizamientos, porque el suelo queda des protegido.

Los deslaves son un tipo de corrimiento de tierra, en los cuales una capa del suelo se separa desde el lecho de roca. En este caso, la tierra de un cerro o gran montículo se desmorona como consecuencia, principalmente, de la lluvia. (Gptsachila, 2015)

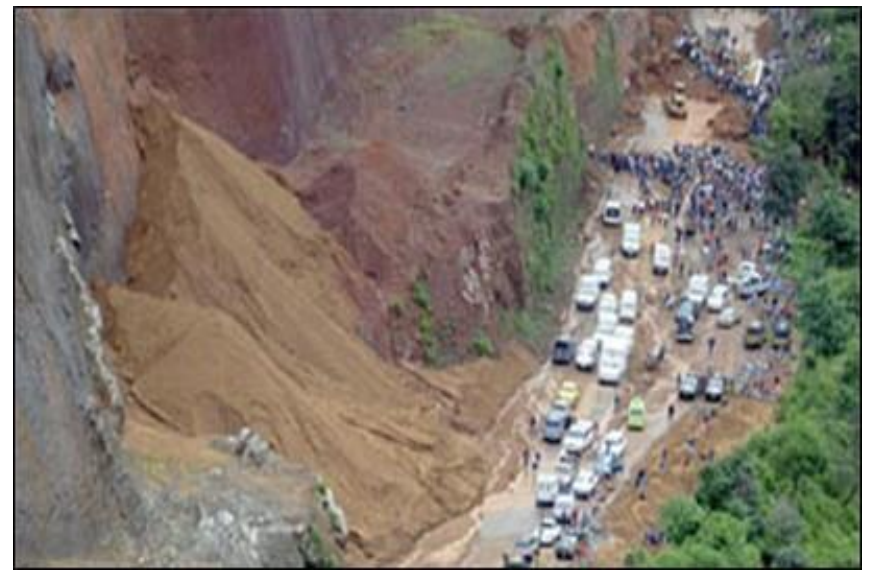

Figura 1. Deslave en carretera. (Saavedra, 2014)

Cuando una masa de tierra, rocas o escombros se mueve por una pendiente hacia abajo, lleva consigo otros residuos saturados de agua que tienden a fluir hacia canales. Son comunes en las líneas costeras, en las costas y mar adentro, detonados por la fuerza de gravedad.

Los deslizamientos pueden producirse a un ritmo lento, de unos cuantos milímetros por año, o producirse de forma muy rápida, a velocidades de hasta 20 millas por hora $(32.1 \mathrm{~km} / \mathrm{h})$. Son altamente peligrosos y pueden convertirse en verdaderos desastres naturales cuando sepultan poblaciones bajo toneladas de tierra. Son muy comunes y en algunas regiones causan más 
problemas que las tormentas o los terremotos. Por ejemplo, en Estados Unidos ocasionan pérdidas de alrededor de 1-3.5 billones de dólares en daños y perjuicios y aproximadamente 2550 muertos por año.

Desafortunadamente, no hay manera de evitar los deslaves puesto que obedecen a fuerzas físicas de la naturaleza, pero sí se pueden prevenir los daños si se aplican prácticas correctas de ingeniería, de investigación geológica y de protección civil. (Gptsachila, 2015)

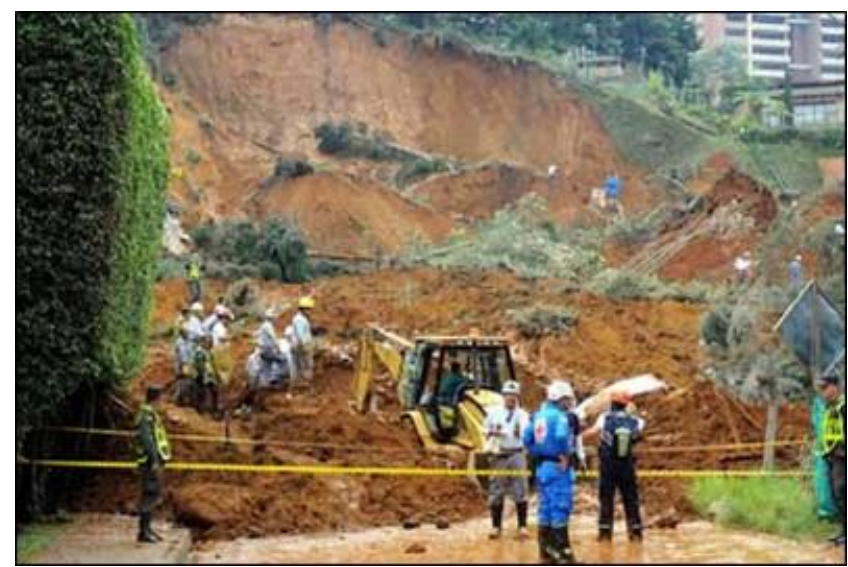

Figura 2. Limpieza de deslave. (Norma, 27)

Se producen deslizamientos cuando capas enteras de terreno se mueven sobre el material firme que tienen por debajo. En su movimiento siguen uno o varios planos de corte del terreno. Los desprendimientos son fragmentos de roca que se separan de un talud y caen saltando por el aire en buena parte de su recorrido. Los aludes son caídas de grandes masas de tierra o nieve.

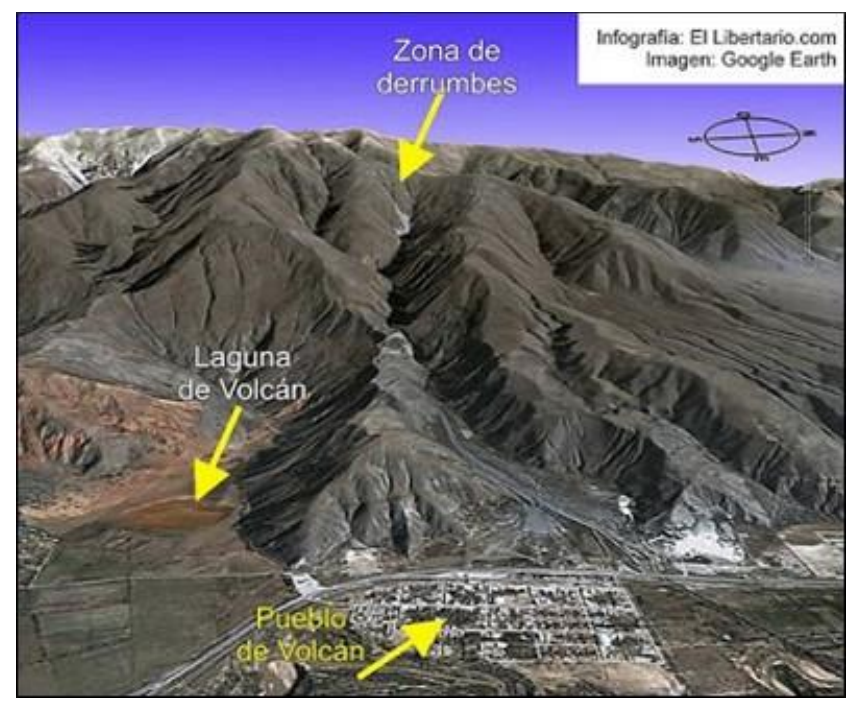

Figura 3. Zonas de derrumbe en una montaña o volcán. (Gestionderiesgos)

Los derrumbes de tierra, también conocidos como deslaves de lodo y aludes, y pueden ser causados por una variedad de factores que incluyen los terremotos, tormentas e incendios. 
Estos fenómenos son desplazamientos de masas de tierra o rocas por una pendiente en forma súbita o lenta. Si bien la gravedad que actúa sobre las laderas es la principal causa de un deslizamiento, su ocurrencia también depende de las siguientes variables:

- Clase de rocas y suelos

-Topografía (lugares montañosos con pendientes fuertes)

- Orientación de las fracturas o grietas en la tierra.

- Cantidad de lluvia en el área.

-Actividad sísmica.

-Actividad humana (cortes en ladera, falta de canalización de aguas, etc.).

-Erosión (por actividad humana y de la naturaleza). (Norma, 27)

Existen dos tipos de deslizamientos o derrumbes. Deslizamientos lentos.- Son aquellos donde la velocidad del movimiento es tan lento que no se percibe. Este tipo de deslizamiento genera unos pocos centímetros de material al año. Se identifican por medio de una serie de características marcadas en el terreno.

Deslizamientos rápidos.- Son aquellos donde la velocidad del movimiento es tal que la caída de todo el material puede darse en pocos minutos o segundos. Son frecuentes durante las épocas de lluvias o actividades sísmicas intensas. Como son difíciles de identificar, ocasionan importantes pérdidas materiales y personales.

Los deslizamientos o movimientos de masa no son iguales en todos los casos, y para poder evitarlos o mitigarlos es indispensable saber las causas y la forma como se originan. Estas son algunas de las formas más frecuentes:

\section{Caída}

Una caída se inicia con el desprendimiento de suelo o roca en una ladera muy inclinada. El material desciende principalmente a través del aire por caída, rebotando o rolando. Ocurre en forma rápida sin dar tiempo a eludirlas.

\section{Volcamiento}

Consiste en el giro hacia delante de una masa de suelo o roca respecto a un punto o eje debajo del centro de gravedad del material desplazado, ya sea por acción de la gravedad o presiones ejercidas por el agua. 


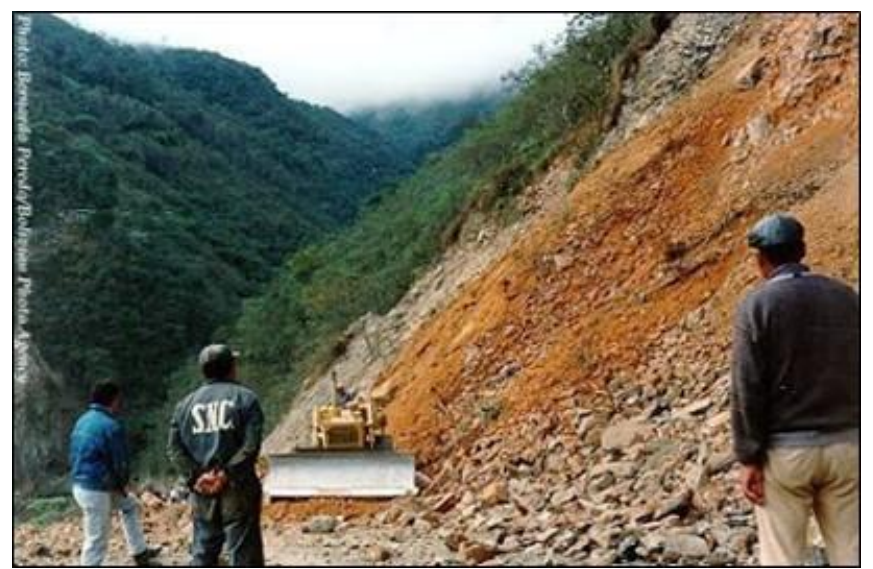

Figura 4. Deslizamiento de tierra en ladera. (Geoenciclopedia)

\section{Deslizamiento}

Es el movimiento, hacia abajo de una ladera, de una masa de suelo o roca el cual ocurre principalmente sobre una superficie de ruptura o falla (debilidad del terreno) y se puede presentar de dos formas: (Yepes, 2015)

Deslizamiento Rotacional: Los desplazamientos ocurren o tienen lugar a lo largo de una superficie de ruptura de forma curva o cóncava.

Deslizamiento Traslacional: Consiste en el desplazamiento de una masa a lo largo de una superficie de ruptura de forma plana u ondulada.

Flujos de tierra. Son movimientos lentos de materiales blandos. Estos flujos frecuentemente arrastran parte de la capa vegetal.

Flujos de lodo. Se forman en el momento en que la tierra y la vegetación son debilitadas considerablemente por el agua, alcanzando gran fuerza cuando la intensidad de las lluvias y su duración es larga.

Reptacion. Es la deformación que sufre la masa de suelo o roca como consecuencia de movimientos muy lentos por acción de la gravedad. Se suele manifestar por la inclinación de los árboles y postes, el corrimiento de carreteras y líneas férreas y la aparición de grietas.

\section{Causas de los deslaves}

Las capas del suelo están unidas por variadas fuerzas, y una de ellas es la fricción, una fuerza de resistencia al deslizamiento entre dos superficies. Es justamente lo que ocurre con la tierra, pero si algún elemento se introduce entre las superficies inclinadas se produce un deslizamiento.

Un deslave puede iniciarse en la ladera o en una pendiente pronunciada si la tierra de ésta se satura rápidamente con agua. Las principales causas son las siguientes: 
Lluvias excesivas o intensas. En zonas altamente susceptibles a los deslaves, pueden ocurrir después de una racha corta de lluvia. Agua de deshielo que debilita el suelo. Erosión causada por corrientes de agua. Modificaciones en las aguas subterráneas. Cambios en el nivel del agua. (Gestionderiesgos)

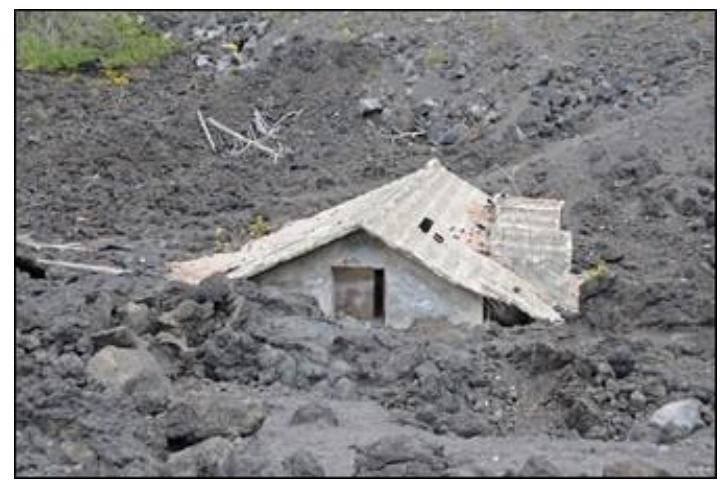

Figura 5. Vivienda sepultada tras un deslave (Saavedra, 2014)

La velocidad del flujo depende de la cantidad de agua, el volumen de la tierra, el ángulo de inclinación y el tipo de tierra.

Algunas zonas son más vulnerables que otras. Por lo general, aquellas cuyo suelo ha sido modificado por las acciones humanas o por los incendios forestales u otros desastres naturales, tienen más probabilidades de sufrir deslizamientos de tierra, así como aquellas pendientes modificadas para la construcción de edificios y las zonas escarpadas situadas en la parte inferior de las montañas o cañones.

En resumidas cuentas, se puede decir que las causas de los deslaves son geológicas, morfológicas y antropogénicas (derivadas de las actividades humanas).

\section{Efectos de los deslaves}

Los deslaves suelen ser muy aparatosos, en el sentido estricto de la palabra. Cuesta abajo, la tierra recoge árboles, autos y construcciones, ocasionando severos daños económicos. Los puentes quedan bloqueados, se fragmentan las líneas eléctricas y las poblaciones quedan incomunicadas.

En el mejor de los casos, los daños económicos son mayores que las vidas que un deslave puede cobrar. Las personas que sobreviven a un desastre de gran magnitud se ven vulnerables ante los riesgos a la salud que entrañan el lodo y los residuos. Si un terreno ha sufrido deslaves, probablemente sufra otros en el futuro. 


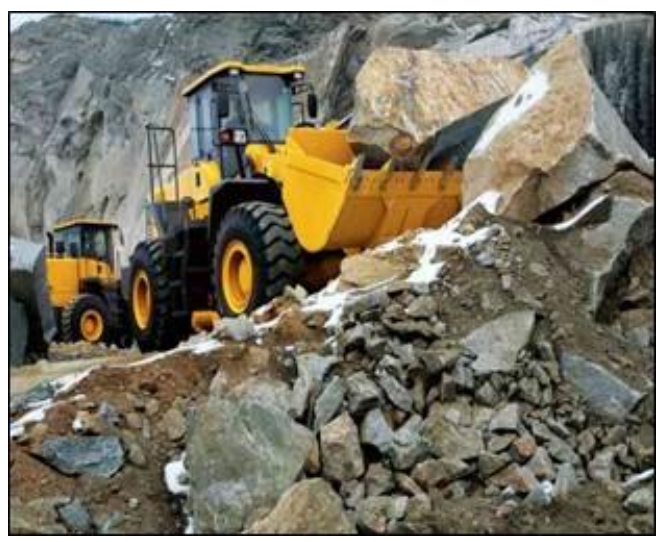

Figura 6. Uso de cargador frontal para la remoción de rocas tras un deslave. (Gptsachila, 2015)

\section{Factores que influyen en la estabilidad de las laderas}

El que una ladera permanezca estable o sufra un deslizamiento depende de la unión de varios factores, entre los que están

Características del terreno.- Los lugares montañosos con pendientes fuertes son los que con más facilidad sufren deslizamientos, aunque en ocasiones pendientes de muy pocos grados son suficientes para originarlos si la roca está muy suelta o hay mucha agua en el subsuelo.

Condiciones climáticas.- En las regiones lluviosas suele haber espesores grandes de materiales alterados por la meteorización y el nivel freático suele estar alto lo que, en conjunto, facilita mucho los deslizamientos. Las lluvias intensas son el principal factor desencadenante de deslizamientos en España.

Macizos rocosos con fallas y fracturas.- Tienen especial importancia en los desprendimientos. En España la mayoría de las caídas de rocas y otros materiales tiene lugar en lugares en los que el terreno tiene abundantes fracturas y se ha ido produciendo erosión en la base de sus laderas. En estos lugares cuando llueve intensamente con facilidad se pueden producir desprendimientos.

Erosión.- Los ríos, el mar u otros procesos van erosionando la base de las laderas y provocan gran cantidad de deslizamientos. En las costas españolas estos fenómenos son muy comunes y provocan el retroceso de los acantilados, sobre todo en las costas del Atlántico, en Canarias y en Baleares.

Expansividad de las arcillas.- Las arcillas tienen la propiedad de que al empaparse de agua aumentan su volumen. Esto supone que los terrenos arcillosos en climas en los que alternan periodos secos con otros húmedos se deforman y empujan taludes, rocas, carreteras, etc. provocando deslizamientos y desprendimientos. (Costes, 1975) 


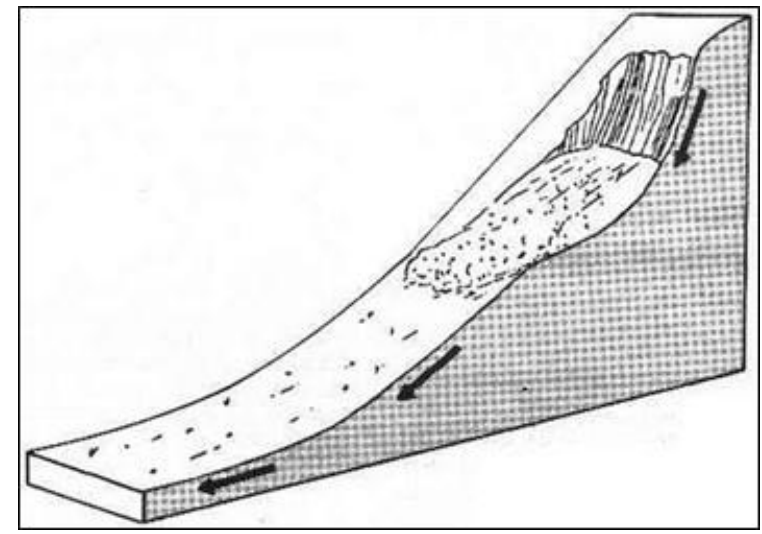

Figura 7. Vista de la zona donde se produce el deslave en la ladera de una montaña. (Costes, 1975)

Acciones antrópicas.- Los movimientos de tierras y excavaciones que se hacen para construir carreteras, ferrocarriles, edificaciones, presas, minas al aire libre, etc. rompen los perfiles de equilibrio de las laderas y facilitan desprendimientos y deslizamientos. Además normalmente se quitan los materiales que están en la base de la pendiente que es la zona más vulnerable y la que soporta mayores tensiones lo que obliga a fijar las laderas con costosos sistemas de sujeción y a estar continuamente rehaciendo las vías de comunicación en muchos lugares.

Se conoce la acción de otros factores como terremotos, rocas calizas (estructuras kársticas), etc., que, en ocasiones, provocan movimientos del terreno, pero cuya importancia es comparativamente menor que los citados anteriormente.

\section{Proceso de remoción de tierra.}

La mecanización de las obras públicas es cada vez mayor, y la repercusión en el precio de las diferentes unidades de obra está muy influenciada por los rendimientos de los equipos empleados, por sus precios horarios y por la eficacia de su utilización. Los costes de la maquinaria acaparan un $42 \%$ del coste de todas las unidades de obra en una carretera. Las unidades que componen el movimiento de tierras en una obra suponen porcentajes importantes del presupuesto total de dichas obras. En una autovía puede suponer entre el 20 y $30 \%$ del coste mientras que en una presa de materiales sueltos, este porcentaje puede subir del 45 al 75\%, según los casos.

Se entiende por movimiento de tierras al conjunto de actuaciones a realizarse en un terreno para la ejecución de una obra. Se denomina excavación a la separación o extracción de determinadas partes de dicho volumen, una vez superadas las fuerzas internas que lo mantenían unido: cohesión, adherencia, capilaridad, etc. Llamamos carga a la acción de depositar los productos de excavación en un determinado medio de transporte. Genéricamente, se puede clasificar la maquinaria utilizada en el movimiento de tierras en los siguientes grupos: (Costes, 1975) 


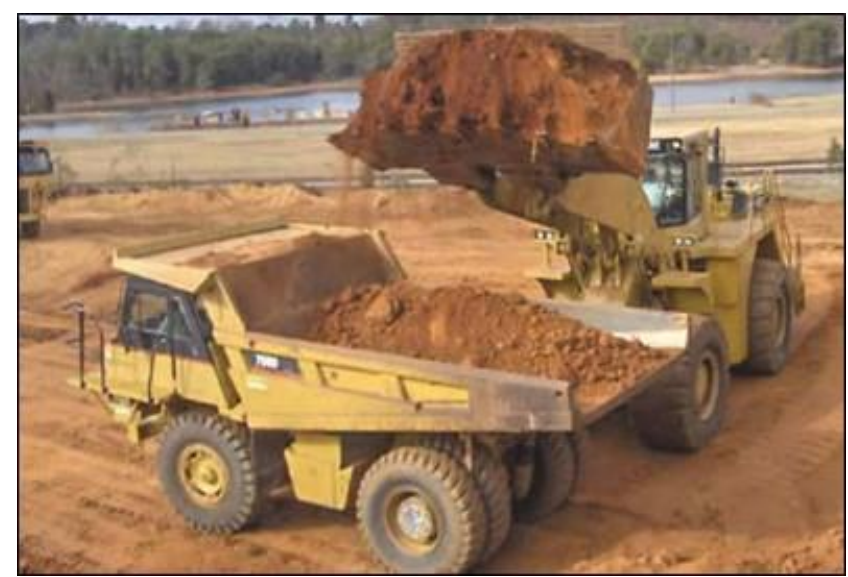

Figura 8. Remoción de tierra y carga hacia la máquina de transporte de material. (Saavedra, 2014)

Equipos de excavación y empuje: son equipos de arranque tales como tractores con palas empujadoras: bulldozer. Equipos de excavación y carga: excavadoras de pala frontal, retroexcavadoras, etc. Equipos cargadores: palas cargadoras. Equipos de excavación y refino: Motoniveladoras, traíllas y mototraíllas. Equipos de acarreo: Camiones volquete, autovolquetes, remolques, camiones góndola, dumpers y motovagones. Equipos de compactación:

Compactadores de ruedas neumáticas, rodillos de "pata de cabra", compactadores vibratorios. Otro tipo de equipos: Cucharas bivalvas, dragalinas, topos, dragas, bombas de succión, etc. Los equipos y medios empleados para la excavación de tierras pueden clasificarse de diversas formas: las que atienden a la traslación de la maquinaria, las que contemplan la resistencia a compresión de los terrenos y las que se refieren a su excavabilidad. (Geoenciclopedia)

Según el modo de trasladarse, se clasifican en: Máquinas que excavan y trasladan la carga: tractores con hoja empujadora o con escarificador, motoniveladoras, mototraíllas y palas cargadoras. Efectúan la excavación al desplazarse, o bien, como la pala cargadora, excava y luego traslada la carga.

Máquinas que excavan situadas fijas, sin desplazarse: palas excavadoras hidráulicas o de cables, dragalinas, excavadoras de rueda frontal o de cangilones, dragas de rosario y rozadoras. Cuando la excavación a realizar sale de su alcance, se debe trasladar a una nueva posición de trabajo, si bien no excava durante el desplazamiento.

Máquinas especiales: topos, dragas y bombas de succión, dardos y chorros de agua y fusión térmica. La excavación se realiza mediante otros procedimientos distintos a los anteriores.

Para realizar movimiento de tierra, se ejecutan algunas tareas como la etapa de despeje, que incluye limpiar el terreno de arbustos, maleza, basuras, etcétera y luego se procede con el replanteo para comenzar con la excavación. "Lo que se hace en proyectos mineros, es sacar todo el material que no tiene mineral y usarlo para relleno de plataformas donde habrán instalaciones o simplemente llevarlo al botadero. Esa es la etapa de pre-stripping", detalla Larraín. El ingeniero comenta que a veces en algunas faenas también se pide retirar "top-soil", dependiendo de dónde estén ubicadas geográficamente o de los compromisos ambientales adquiridos. 
Dependiendo del material se pueden tener dos tipos de trabajos: en tierra y en roca. Para los primeros, que por lo general se realizan por medios mecánicos, se deben considerar las características del terreno que influyen en el desempeño de la maquinaria correspondiente (cohesión, densidad, compacidad) y también factores intrínsecos como asentamientos, niveles freáticos o zonas plásticas. Respecto a los trabajos en roca, se consideran sus características (dureza, forma, estratificación, etcétera), así como la cantidad y tipos de explosivos a utilizar, en el caso de rocas de gran dureza.

Etapas del proceso

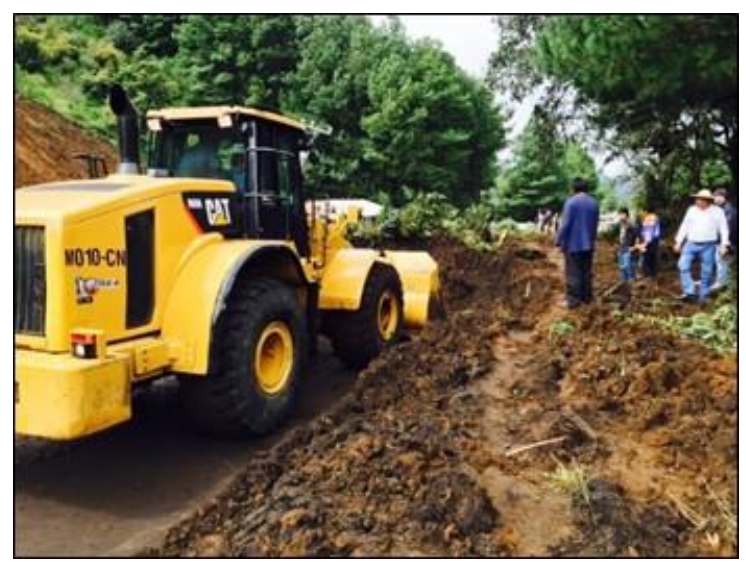

Figura 9. Etapa de despeje previo al movimiento de tierra con a cargadora frontal. (Yepes, 2015)

Si bien no es una fase del proceso en sí, para trasladar la maquinaria que se utilizará, generalmente circula una camioneta por delante como escolta, indicando que va una carga ancha. Detrás de los equipos circula otra camioneta que se encarga de evitar un acercamiento muy próximo o que intenten adelantarlos sin previa coordinación con la escolta anterior.

Una vez en el lugar determinado, se da paso a las fases de explotación y pre-stripping y luego se avanza a las zonas de mineral propiamente tal. "La programación se hace de acuerdo a cómo va la veta de extracción y capacidad de proceso de las minas. Son programaciones hechas con software topográficos que van indicando las cantidades a explotar, de dónde tienes que sacar y qué cantidad (plan minero), y a qué lugar hay que ir a botar el material”, cuenta Larraín, agregando que la extracción en rajo abierto es diferente a la de túneles porque en estos logras una extracción más precisa, mientras que en los primeros el mineral es más difícil de homogenizar.

La siguiente etapa se refiere a la de carguío, donde los expertos coinciden en que es fundamental un diseño eficiente en la que la operación trabaje de manera integrada con los camiones, equipos ampliamente utilizados en los procesos realizados en rajo abierto, gracias a su mayor flexibilidad para realizar grandes movimientos de tierra.

Si bien, no hay un tipo de carga ideal, las palas de cable son muy apreciadas gracias a su robustez y efectividad de costo. Las grandes excavadoras hidráulicas, también se han transformado en una buena alternativa para estas actividades. (Geoenciclopedia) 


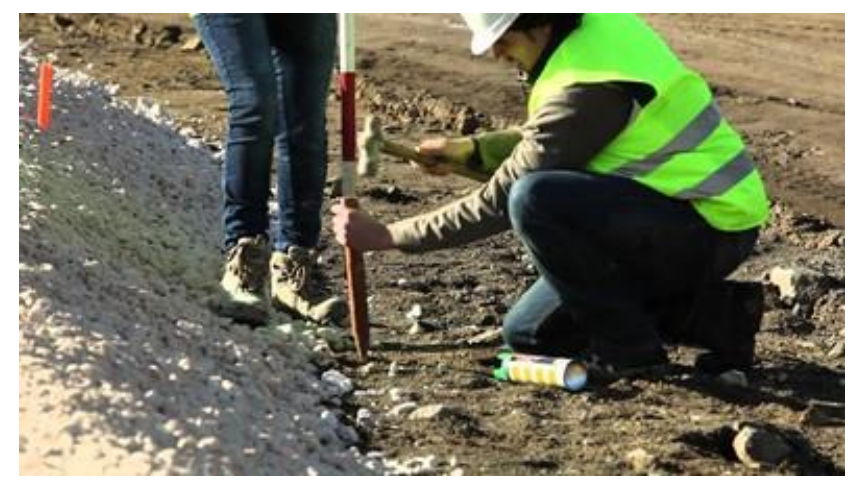

Figura 10. Etapa de replanteo. (Costes, 1975)

En el caso de movimientos de volúmenes pequeños de material, se utilizan excavadoras hidráulicas y cargadores frontales, ya que el bajo costo de capital y la movilidad, ayudan a optimizar el sistema de carguío.

En la etapa de transporte, se incluye el diseño de la ruta, cuyos criterios más importantes se refieren a la distancia de detención, señales de distancia y velocidad, los que cobran mayor relevancia cuando hay intersecciones, curvas y ubicaciones de descarga. En cuanto a la construcción de las rutas estas contienen material de sub-base y pavimentos de calidad y espesor adecuados (dependiendo en parte del proyecto). Para aumentar su seguridad y productividad se utilizan caminos duros, lisos y con buena tracción. "A los caminos se les hace mantenimiento. Hay equipos que van sacando las piedras y los van dejando lo más parejos posible”, señala Larraín, agregando que uno de los temas relevantes dentro del presupuesto, es que las rutas duren lo más posible ya que las piedras son agentes que desgastan mucho a los vehículos. El experto indica que para mantener los caminos se usan motoniveladoras, rodillos y camiones aljibe que van lanzando agua para mantener las superficies húmedas y regadas.

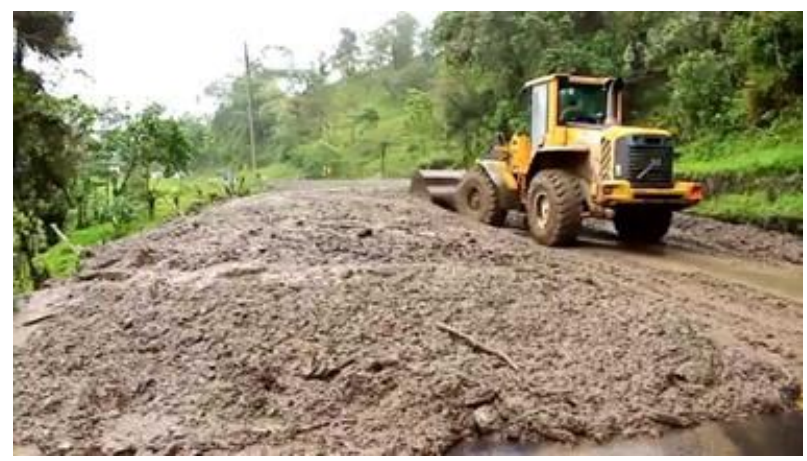

Figura 11. Extendido del terreno. (Yepes, 2015)

\section{Uso de máquinas}

Como se mencionó anteriormente, las principales máquinas utilizadas para movimiento de tierra son excavadoras, cargadoras (también llamadas cargadora de pala frontal o pala cargadora frontal) y los grandes camiones que trasladan el material de un lugar a otro. En el caso de las primeras, como su nombre lo indica, se usan para realizar excavaciones, donde el equipo se posiciona sobre el terreno y con una "cuchara" arranca el material que arrastra y deposita en su interior, gracias a un movimiento que va desde abajo hacia arriba. Dentro de las variantes de 
esta máquina está la retroexcavadora que a diferencia de la anterior realiza la excavación de arriba hacia abajo y es utilizada para trabajar el movimiento de tierras a nivel inferior al plano de apoyo o un poco superior a este.

Por su parte, las cargadoras, son palas mecánicas montadas sobre ruedas o cadenas. Su estructura de soporte posee dos brazos articulados que se accionan de manera hidráulica. Ampliamente utilizadas en los procesos de movimiento de tierra están las palas de cable, palas hidráulicas y cargadores frontales, donde las primeras se prefieren para grandes tasas de producción desde carbón muy suave hasta mineral duro, mientras que para aplicaciones de menor escala se opta por el cargador frontal. "Para la carga debe haber un balance, ya que se recomienda cargar cuatro o cinco paladas. Lo ideal es que con cinco ciclos de carguío el camión deba irse, por tanto el tamaño de la pala debe ser acorde con eso", comenta Larraín. (Norma, 27)

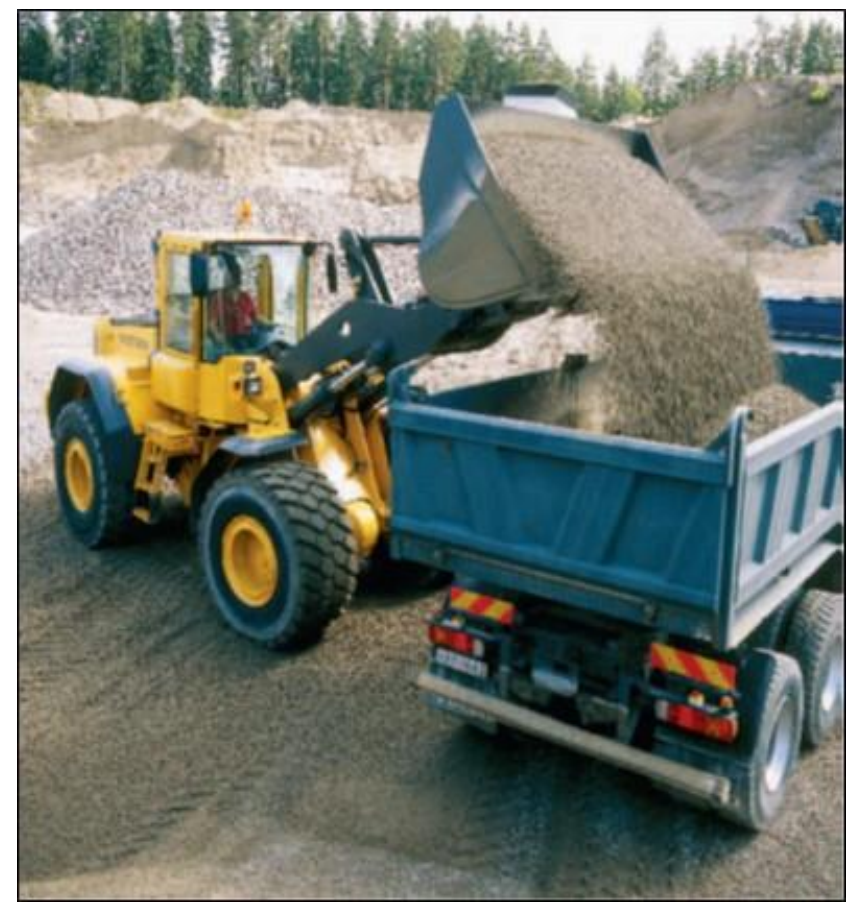

Figura 12. Etapa de carga y descarga del material retirado en el camión de transporte. (Volvo Construction Equipment)

\section{Características de la Cargadora frontal sobre ruedas L120E Volvo}

La nueva máquina cargadora de Volvo de 20 toneladas es potente y de fácil operación. La bien conocida L120 se ha convertido en la L120E, que es más rápida, más eficaz y más productiva que antes. El operador se mantiene descansado y concentrado durante todo el turno de trabajo y la máquina trabaja además produciendo los menores impactos posibles en el medio ambiente. Operar la L120E es todo un placer. 


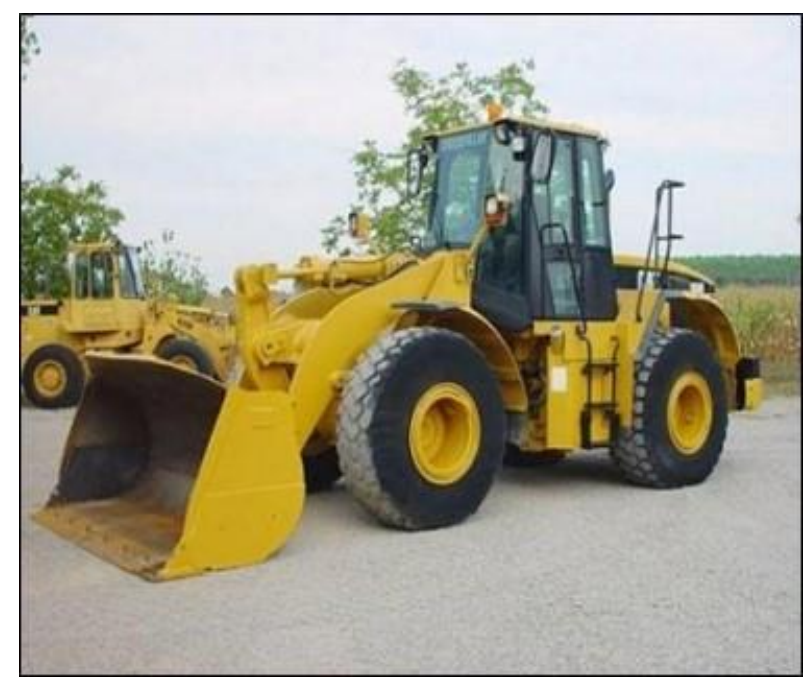

Figura 13. Cargadora frontal sobre ruedas L120E Volvo. (Volvo Construction Equipment)

Al maniobrar la L120E se tiene la sensación de estar al volante de un camión moderno más que de una potente máquina cargadora. Sin embargo, se trata precisamente de eso, de una máquina cargadora con recursos impresionantes. En colaboración con la hidráulica sensible a la carga, su motor de altas prestaciones y bajas emisiones desarrolla en cada momento la potencia necesaria, evitándose que el aceite circule por el sistema de forma innecesaria.

La patentada cinemática TP, cucharas y garras bien adaptadas y un amplio surtido de implementos hacen de la L120 una cargadora de gran productividad en canteras de grava, en arenales y en puertos para cargar en barcos y vagones de tren. También es perfecta para la manipulación de astillas de madera en la industria papelera y una excelente máquina maderera que descarga un camión con troncos a la misma velocidad que una máquina de mayor tamaño.

\section{Fuerte y versátil}

La nueva L120E es potente, versátil y muy fácil de maniobrar. El operador está cómodamente sentado y tiene pleno control sobre la máquina y el trabajo. El motor y la hidráulica responden de forma directa y exacta. El operador dispone de una visibilidad excepcional alrededor de la máquina y de aire puro y limpio en la cabina. Que la máquina sea tan cómoda y fácil de maniobrar hace también que el operador pueda trabajar durante más tiempo sin cansarse. Tanto el operador como la máquina pueden producir más, día tras día, semana tras semana.

\section{Excelente ahorro general de costes}

La excepcional productividad y seguridad de funcionamiento, la provechosa financiación, el bajo consumo de combustible y el alto valor de reventa contribuyen a un gran ahorro general de costes. La disponibilidad de la máquina es, además, excepcionalmente alta gracias a las mínimas necesidades de servicio de la misma y a la sencillez y rapidez con la que se efectúa el mantenimiento diario. A esto pueden añadirse las extraordinarias características de operación que aumentan todavía más la productividad. Todo ello dota a la L120E de una excelente economía 
tanto a corto como a largo plazo. Calcule como calcule, con la L120E hará el mejor negocio. (Volvo Construction Equipment)

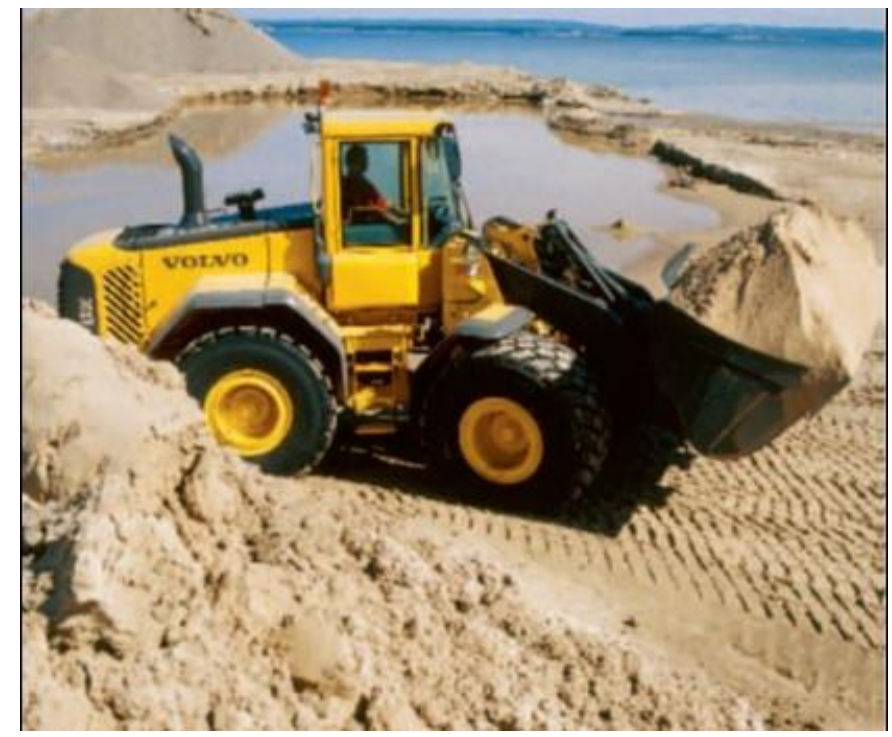

Figura 14. Gran desempeño y versatilidad. (Volvo Construction Equipment)

La nueva Volvo L120E es una máquina sumamente productiva. El potente motor de bajo régimen y el cambio automático de marchas proporcionan una respuesta extraordinaria incluso en las más duras tareas. Los ejes están óptimamente conjuntados con la totalidad de la línea motriz. El resultado es una productividad elevada, muy bajo consumo de combustible y una superior economía de operación.

El motor Volvo D7D de control electrónico proporciona respuesta y ciclos de trabajo más rápidos El nuevo motor de 7 litros cuenta con unas características extraordinarias gracias a la nueva unidad de mando que proporciona siempre una dosificación óptima del combustible. La máquina responde potentemente con excepcional fuerza tanto en la hidráulica como en la línea motriz. Las emisiones de gases de escape son mínimas y la L120E proporciona además un gran ahorro de combustible. La combinación de todo esto se traduce en una superior productividad y economía, tanto a corto como a largo plazo.

Cambio automático dependiente del régimen y la velocidad La transmisión del tipo de contra ejes, desarrollada por Volvo, proporciona las maniobras de cambio más suaves posibles. El operador sólo tiene que elegir entre marcha adelante, marcha atrás o kickdown, y el APS elegirá siempre la marcha adecuada según el régimen del motor, la velocidad de la máquina y el programa de conducción seleccionado por el operador. Proporciona el máximo de fuerza posible y el más bajo consumo de combustible, independientemente de las condiciones de trabajo. 


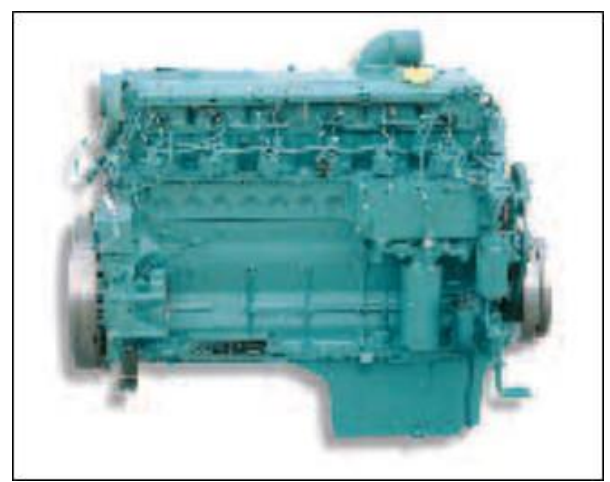

Figura 15. Motor Volvo D7D (Volvo Construction Equipment)

Un entorno de trabajo cómodo y seguro aumenta el bienestar y la productividad del operador. Por eso hemos dedicado tanto trabajo a que la cabina sea lo más agradable y cómoda posible. La cabina Care Cab confirma el liderazgo de Volvo en lo referente al entorno del operador y el confort en la cabina. Care Cab. Un puesto de trabajo limpio y confortable Con un buen clima en la cabina, el operador se mantiene descansado durante toda la jornada. Todo el aire se filtra a través de dos filtros para ofrecer el entorno de cabina más limpio del mercado. El aire pasa primero por el pre filtro y es purificado a continuación a través del filtro principal. Además, el eficaz acondicionador de aire proporciona una temperatura agradable durante todo el año, independientemente de la temperatura exterior. Un puesto de trabajo cómodo que fomenta la productividad Hay una gran variedad de asientos para elegir, con muchas posibilidades de ajuste para máximo confort individual. Los instrumentos quedan claramente visibles. Toda la información importante está centralizada en el campo visual del operador.

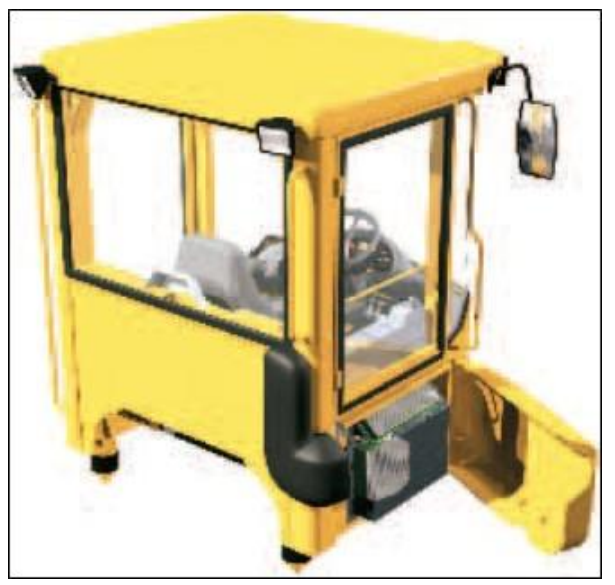

Figura 15. Cabina CareCab de la cargadora frontal volvo. (Volvo Construction Equipment)

\section{Materiales y Métodos}

Los materiales y métodos utilizados para la limpieza y remoción de tierra por deslaves en la vía Aloag-Santo Domingo ser conformes con las normas nacionales o, cuando sea posible, con su transposición a las normas europeas, o dependerán de la aprobación de los técnicos europeos o, en ausencia de éstos, los componentes y los materiales deben cumplir los requisitos del proyecto. 
Para nuestro caso emplearemos, a cargadora frontal volvo L120E, las volquetas necesarias para el transporte del material removido, y el personal capacitado para realizar el planteo y el control del proceso de remoción y limpieza de deslave, a continuación se detalla la ficha técnica de la cargadora frontal:

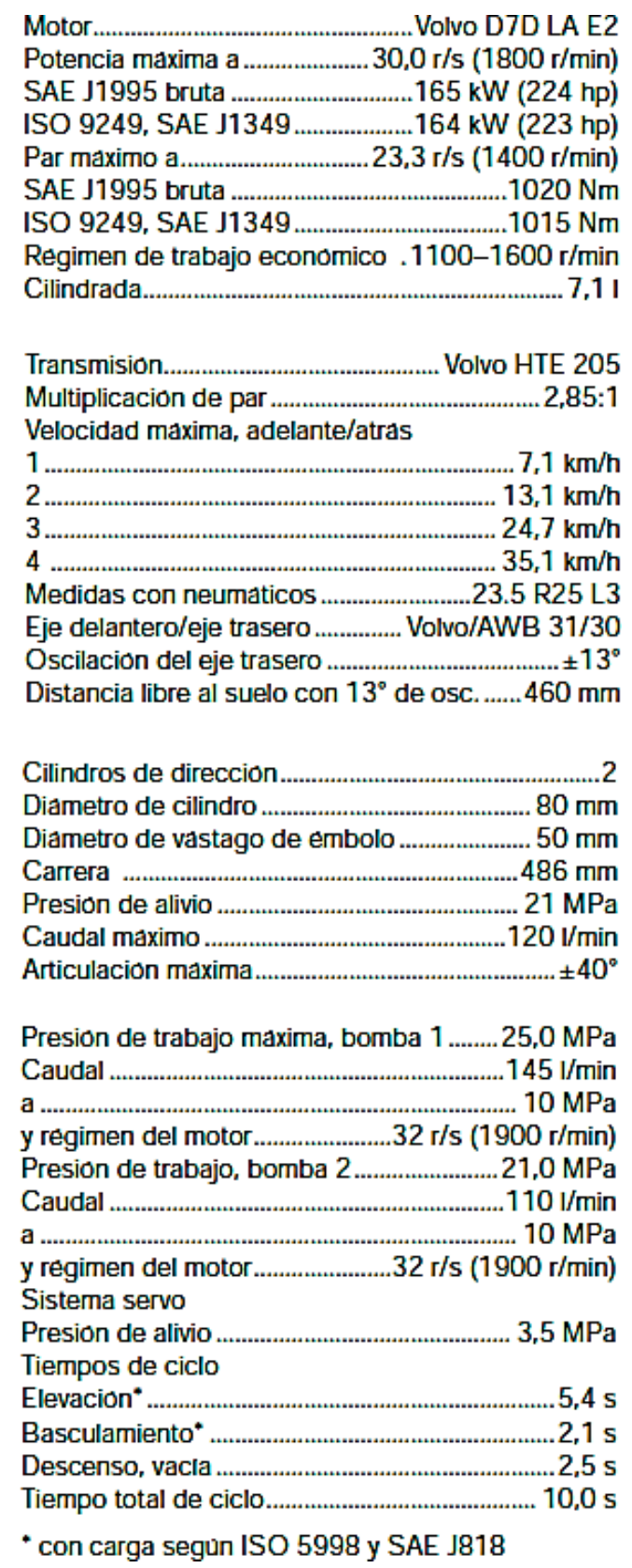

Figura 16. Ficha técnica Volvo L120E (Volvo Construction Equipment)

\section{Método Práctico}

Para determinar que método usar para la elección de la máquina para levar a cabo la remoción y limpieza de os deslaves, se ha escogido e método practico, ya que la práctica que han 
tenido os ingenieros civiles con esta máquina han dado buenos resultados y cumple de una manera óptima y eficaz la operación que se espera cumplir en la obra.

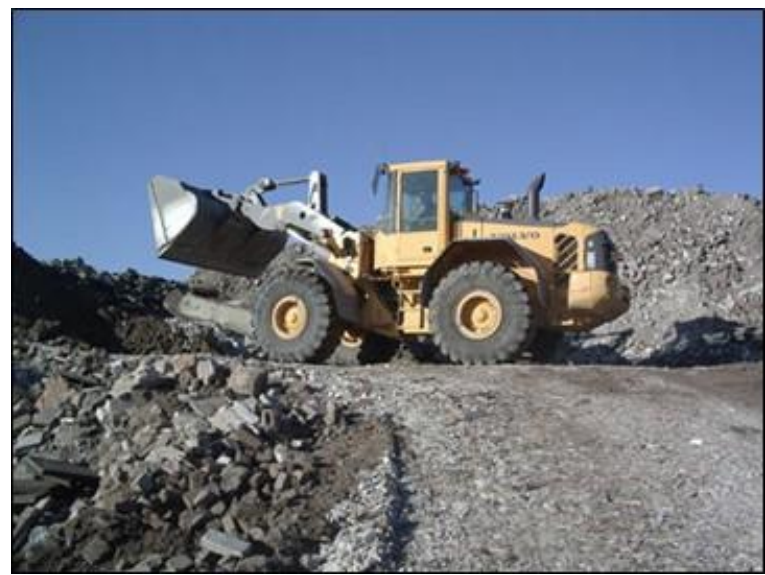

Figura 17. Método practico de ensayo de terreno y remoción de piedras. (Volvo Construction Equipment)

\section{Diseño en 2D y 3D de la Cargadora Frontal en AutoCAD.}

Detallo en este apartado e proceso básico que lleve a cabo para realizar el modelado 3D y de esta manera obtener as vistas 2D de mi máquina, para realizarlo en 3D se procedió a utilizar e programa Sketchup ya que es más didáctico y sencillo y da mejores resultado que al utilizar AutoCAD.

\section{Proceso de modelado en 3D}

El primer paso será realizar el chasis y cabina de la máquina.

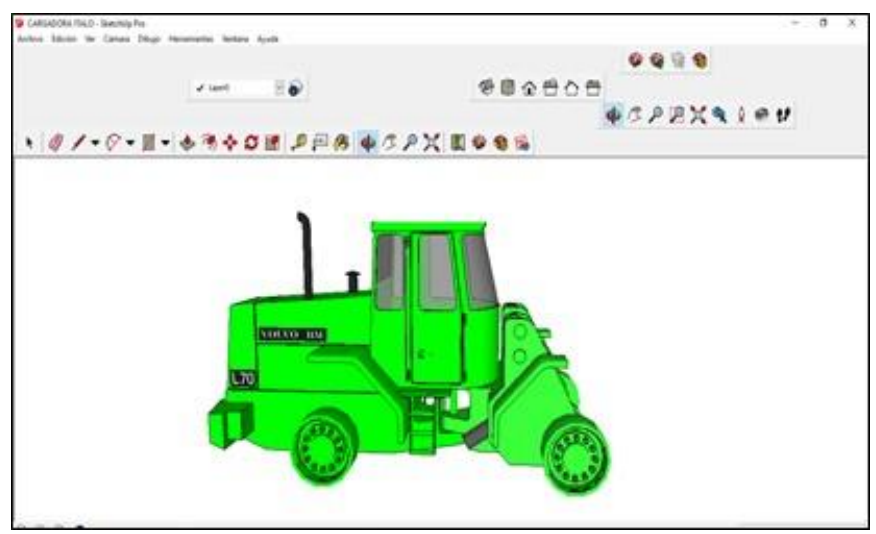

Figura 18. Modelado de chasis y cabina (Volvo Construction Equipment)

Seguido de ello procedí a agregarle las ruedas y la pala, y así termine mi modelado 3D de la máquina. 


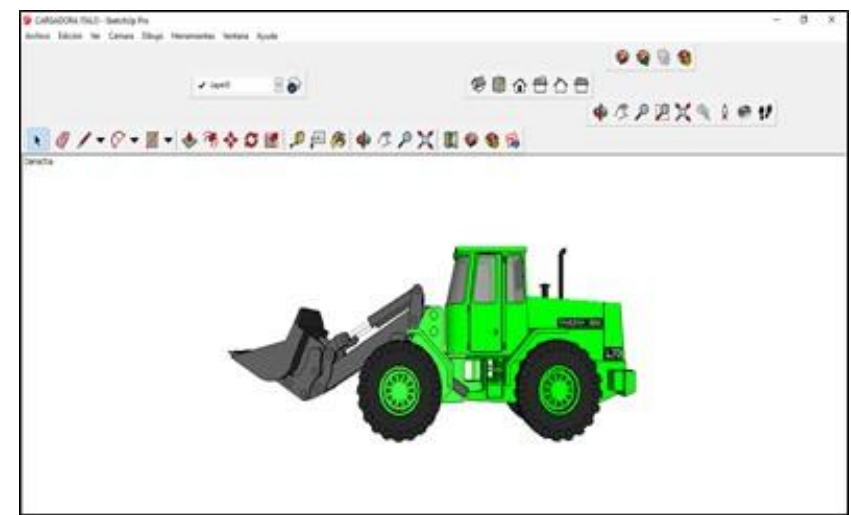

Figura 19. Modelado terminado de la máquina. (Volvo Construction Equipment)

Después de terminar el modelado 3D, el programa Sketchup nos da la opción de exportar una por una las vistas de la máquina, nos brinda el formato .DWG que es soportado por AutoCAD y de esta manera lo podremos editar en ese programa.

\section{Diseño 2D}

Es muy sencillo este proceso ya que una vez exportada la vista, solo tendremos que abrir una por una en AutoCAD y de esta manera haremos los retoques que queramos y podremos agregar las cotas.

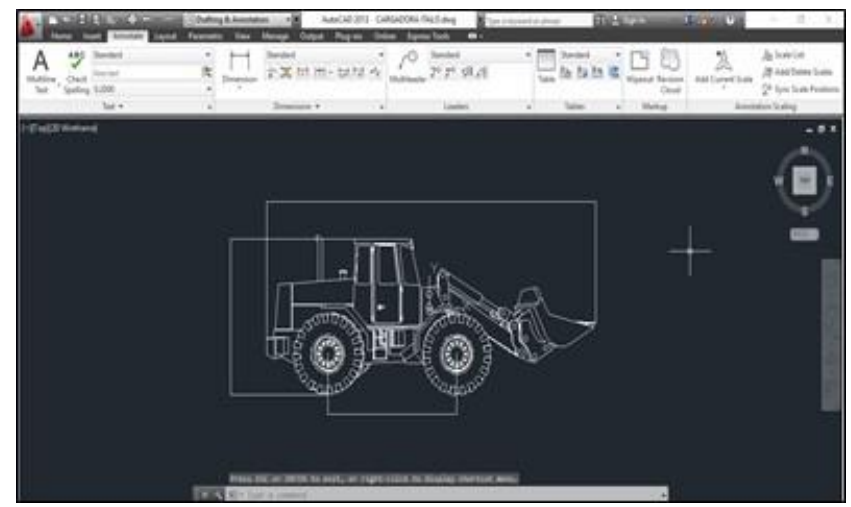

Figura 20. Modificando la vista 2D de la máquina. (Volvo Construction Equipment)

\section{Propósito}

\section{Limpieza y remoción de tierra por deslaves en la vía Aloag-Santo Domingo}

Cada cierto tiempo a lo largo de esta carretera se producen deslaves y deslizamientos de tierra o piedra, para ello es necesario su remoción y limpieza, debido a que es una vía muy transitada y el no contar con ella, ocasiona un tráfico tremendo ya que es una de los ejes viales importantes. En la siguiente figura veremos donde son las zonas que hay los taludes y en donde se producen los deslaves. 


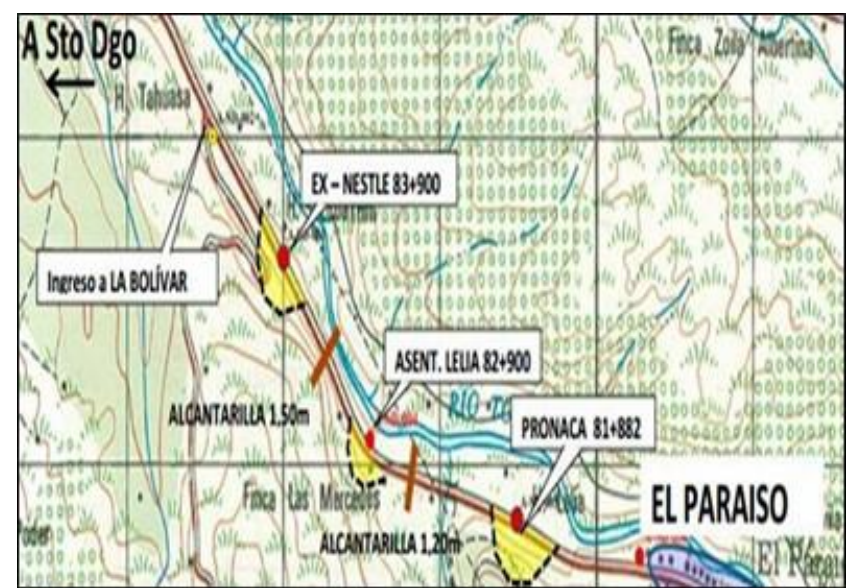

Figura 21. Taludes en la vía a Quito, tramo La Unión del Toachi- Santo Domingo. (Gptsachila, 2015)

\section{Resultados y Discusión}

Luego de detallar los procedimientos que se llevan a cabo para la limpieza y remoción de tierra como técnica, y las características de la máquina que se va a usar como herramienta, veremos los resultados que se ha obtenido una vez implementada la maquinaria.

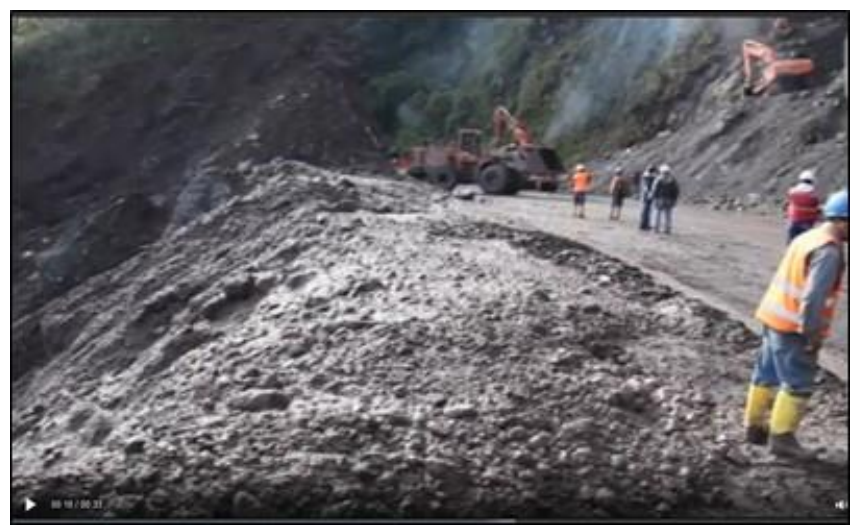

Figura 26. Implementación de la cargadora frontal para limpieza y remoción de tierra en el deslave de la vía Aloag-Santo Domingo. (Gptsachila, 2015)

Observando la figura nos damos cuenta que ha sido correcta a elección de esta maquinaria, ya que ha ayudado a despejar y remover la tierra que cubría la carretera de esta carretera luego de que se ha presentado el deslave.

\section{Conclusiones}

Al termino de mi artículo y una vez analizada toda la información expuesta, puedo concluir que el uso de una cargadora frontal de la que he seleccionado para su implantación en la limpieza y remoción de tierra es una aplicación correcta de esta maquinaria, ya que se ha cumplido con el propósito y de una manera eficiente y se han ahorrado recursos que pueden ser destinado a otros proyectos civiles para el bienestar de todo los ciudadanos. 
Se deberá capacitar al personal para que pueda ejecutar una correcta operación de esta maquinaria, y además de indicarle todas las normas de seguridad en la operación no solamente de la maquinaria, sino de la actividad en general que realice durante esta obra, ya que es muy riesgosa porque se podría presentar nuevos deslaves mientras se esté realizando la limpieza de sitio.

\section{Bibliografía}

Costes, J. (1975). Máquinas para movimiento de tierras: descripción, utilización, entretenimiento, Barcelona: Editores Tecnicos Asociados, S.A.

Geoenciclopedia, «geoenciclopedia.com,» [En línea]. Available: http://www.geoenciclopedia.com/deslave/. [Último acceso: 0102 2016].

Gestionderiesgos, «gestionderiesgos.gob.ec,» [En línea]. Available: http://www.gestionderiesgos.gob.ec/deslaves/. [Último acceso: 0102 2016].

Gptsachila, «gptsachila.gob.ec,» $07 \quad 04 \quad 2015 . \quad$ [En línea]. Available: http://www.gptsachila.gob.ec/index.php/7-blog/58-se-buscan-soluciones-mas-integralespara-la-via-aloag-santo-domingo. [Último acceso: 0102 2016].

Norma, «cuandolatierrasemueve.blogspot.com,» Plantilla Awesome Inc., (2010). [En línea]. Available: http://cuandolatierrasemueve.blogspot.com/2010/10/derrumbes.html. [Último acceso: 0102 2016].

Saavedra, A. «construccionminera.cl,» (2014). [En línea]. Available: http://www.construccionminera.cl/caracteristicas-generales-del-proceso-movimiento-detierra-en-faenas-mineras/. [Último acceso: 0102 2016].

Volvo Construction Equipment, «evocon.rs,» [En línea]. Available: http://www.evocon.rs/pdf/bageri/bageri_tockasi/EW230C.pdf. [Último acceso: 2001 2016].

Yepes, V. «procedimientosconstruccion.blogs.upv.es,» (2015). [En línea]. Available: http://procedimientosconstruccion.blogs.upv.es/category/excavaciones-yvoladuras/movimiento-de-tierras-generalidades/. [Último acceso: 0102 2016]. 